\title{
All-trans retinoic acid restored the osteogenic ability of BMP9 in osteosarcoma through the p38 MAPK pathway
}

\author{
NIAN WU ${ }^{1,2}$, RUIDONG LI ${ }^{1}$, ZIJUN MENG ${ }^{1}$, MAO NIE ${ }^{1}$, QIANZHAO CHEN ${ }^{2,3}$, \\ BAICHENG HE ${ }^{2,3}$, ZHONGLIANG DENG ${ }^{1}$ and LIANGJUN YIN ${ }^{1}$ \\ ${ }^{1}$ Department of Orthopaedics, The Second Affiliated Hospital of Chongqing Medical University; \\ ${ }^{2}$ Chongqing Key Laboratory of Biochemistry and Molecular Pharmacology, \\ Chongqing Medical University; ${ }^{3}$ Department of Pharmacology, \\ School of Pharmacy, Chongqing Medical University, Chongqing, P.R. China
}

Received November 16, 2016; Accepted February 20, 2017

DOI: 10.3892/ijo.2017.3910

\begin{abstract}
Osteosarcoma (OS) is the most common malignant bone tumour and is considered to be a disease caused by a dysfunction in differentiation. Bone morphogenetic protein 9 (BMP9) is the most potent osteogenic factor in mesenchymal stem cells, but it cannot induce osteogenic differentiation in OS cells; this might be one of the determinants in the pathogenesis of OS. All-trans retinoic acid (ATRA) can induce osteogenic differentiation of OS cells and potentiate BMP9-induced osteogenesis in preadipocytes. However, the concomitant effect of ATRA and BMP9 in OS cells is unclear; therefore, in the present study, we focused on this topic. The results showed that BMP9 significantly promoted the proliferation of human OS 143B cells and did not induce osteogenic differentiation of cells in vitro $(\mathrm{p}<0.01)$. ATRA inhibited proliferation and induced osteogenesis in 143B cells; these effects could be enhanced by BMP9 overexpression $(p<0.05)$. ATRA could significantly increase the level of phosphorylated p38 MAPK (p-p38) in 143B cells, while BMP9 did not have any significant effect. Notably, BMP9 overexpression enhanced the ability of ATRA to increase the levels of p-p38. Both the osteogenic differentiation and the anti-proliferative activity of BMP9 in the presence of ATRA decreased upon treatment with a specific inhibitor of p38 MAPK (SB203580) $(\mathrm{p}<0.01)$. This study indicates that the osteogenic differentiation ability of BMP9 in 143B cells can be restored by ATRA, and the combination of BMP9 and ATRA generated a stronger anti-proliferative effect on 143B cells than ATRA alone. This result may be due to the activation of the p38 MAPK pathway.
\end{abstract}

Correspondence to: Dr Liangjun Yin, Department of Orthopaedics, The Second Affiliated Hospital of Chongqing Medical University, 76 Linjiang Road, Yuzhong, Chongqing 400010, P.R. China

E-mail: albertyin.21@163.com

Key words: osteosarcoma, morphogenetic protein 9, all-trans-retinoic acid, osteogenic differentiation, p38 MAPK

\section{Introduction}

Osteosarcoma (OS) is the most common malignant bone tumour, and it mainly affects children and adolescents (1-3). The progression of this disease is characterized by aggressive tumour growth, frequent recurrence, and high risk of pulmonary metastasis $(3,4)$. Although its treatments have advanced from amputation to complex limb-sparing surgery (LSS) and incorporated multi-agent chemotherapy (5), the 5 -year survival rate is only $60-70 \%$ (6). Moreover, the serious side effects associated with traditional chemotherapy drugs greatly decrease the patients' quality of life while reducing the effectiveness of the OS treatments (5). Increasing evidence suggest that OS may be a differentiation dysfunction disease caused by defects in the terminal differentiation of osteoblasts (7-13). Therefore, the promotion and/or circumvention of differentiation defects may be used as an adjuvant therapy for OS.

Bone morphogenetic proteins (BMPs) are multifunctional growth factors, which belong to the TGF- $\beta$ superfamily (14). BMPs play important roles in development and cellular physiology processes, such as proliferation, differentiation, apoptosis, adhesion and migration $(15,16)$. Yang et al suggested that OS cells might be maintained in an undifferentiated state secondary to impaired TGF- $\beta /$ BMP signalling (13). BMP9, a member of BMPs, is known as the most potent osteogenic factor compared to the other family members in mesenchymal stem cells (MSCs) $(17,18)$. However, Luo et al reported that BMPs (including BMP9) were unable to induce bone formation in twelve OS cell lines (including 143B cells) and promoted OS cell growth by locking the cells in the early proliferative phase of the osteogenic pathway (10). Other studies also indicated that BMP9 failed to induce osteogenic differentiation in OS (8,9,11-13). Aberrant expression of BMP9 might result in many human tumours, such as colon cancer (19), breast cancer (20), ovarian cancer (21), hepatocellular carcinoma (22) and gastric cancer (16). These data indicate that the abnormal osteogenic differentiation related to BMP9 might be one of the determinants in the pathogenesis of OS. Therefore, restoring the normal osteogenic ability of BMP9 in OS cells might contribute to the treatment of OS. 
All-trans retinoic acid (ATRA) is a highly potent derivative of vitamin $\mathrm{A}$, which is required for virtually all essential physiological processes and functions (23). The biological effects of ATRA are mediated by two families of nuclear receptors, retinoic acid receptor (RAR) and retinoid $\mathrm{X}$ receptor (RXR), which work as RAR/RXR heterodimers and bind to retinoic acid response elements in the promoter regions of retinoid-responsive genes (24). ATRA can induce differentiation in acute promyelocytic leukaemia (APL) and other tumour types, such as neuroblastoma, breast cancer and melanoma (25-28). Additionally, ATRA can induce osteoblastic differentiation of osteosarcoma cells both in vivo and in vitro (29,30). Luo et al (31) and Ying et al (32) have reported that ATRA-induced osteogenic differentiation is mediated by the retinoid-suppressed phosphorylation of RAR $\alpha$ in OS cells. These findings indicate that RAR $\alpha$ plays a key role in ATRAinduced osteogenic differentiation. It has been reported that ATRA could potentiate BMP9-induced osteogenesis in 3T3-L1 preadipocytes (33). However, the effect of ATRA on the osteogenic ability of BMP9 in OS cells is still unclear.

This study investigated the combined effect of BMP9 and ATRA on proliferation and osteogenic differentiation of human OS 143B cells. The results showed that, in 143B cells, the osteogenic ability of BMP9 could be exerted in the presence of ATRA, and the combination of BMP9 and ATRA generated a stronger anti-proliferative effect than ATRA alone. Additionally, these effects may originate from the activation of the p38 MAPK pathway.

\section{Materials and methods}

Reagents and antibodies. ATRA was obtained from Sigma-Aldrich (St. Louis, MO, USA), dissolved in dimethyl sulfoxide (DMSO), divided to aliquots and stored at $-20^{\circ} \mathrm{C}$. DMSO was used as a control. Antibodies were obtained from Santa Cruz Biotechnology. SB203580 was purchased from Selleckchem (Houston, TX, USA). All other reagents were purchased form Sigma-Aldrich or Thermo Fisher Scientific, unless otherwise indicated.

Cell lines and culture. The human 143B (OS) and HEK293 cell lines were purchased from the American Type Culture Collection (ATCC). 143B cells or HEK293 cells were maintained in Dulbecco's modified Eagle's medium (DMEM; Hyclone) and supplemented with $10 \%$ heat-inactivated FBS (Hyclone Laboratories), $100 \mathrm{U} / \mathrm{ml}$ benzylpenicillin, and $100 \mathrm{mg} / \mathrm{ml}$ streptomycin at $37^{\circ} \mathrm{C}$ in $5 \% \mathrm{CO}_{2}$.

Construction of recombinant adenoviruses. Recombinant adenoviruses expressing BMP9 (AdBMP9) were generated previously using the AdEasy technology, as described (34). AdBMP9 also expressed GFP as a marker to monitor infection efficiency. Adenoviruses expressing GFP (AdGFP) were used as control.

Crystal violet assay. Cell viability was determined with a crystal violet assay, conducted as previously described (35). Briefly, 143B cells were plated in a 24-well plate and treated with the indicated concentrations of ATRA, and/or AdBMP9 and/or SB203580. The cells were washed carefully 2 times with ice-cold $\left(4^{\circ} \mathrm{C}\right)$ phosphate-buffered saline (PBS); then, the cell viability was assessed upon staining with a $0.2-0.3 \%$ crystal violet formalin solution at room temperature for $20 \mathrm{~min}$. For scanning and quantification, $500 \mu \mathrm{l} /$ well of $20 \%$ acetic acid were added to dissolve the crystal violet, and the plate was shaken for $20 \mathrm{~min}$ at room temperature. The absorbance was detected at $570 \mathrm{~nm}$.

Cell cycle analysis. 143B cells were plated into 6-well plates. Then, the cells were treated with ATRA and/ or AdBMP9 for $48 \mathrm{~h}$. The cells were washed 3 times with PBS without calcium and magnesium and fixed in $70 \%$ ethanol overnight at $4{ }^{\circ} \mathrm{C}$. The cells were washed twice with ice-cold PBS and centrifuged at $300 \mathrm{x} \mathrm{g}$ for $10 \mathrm{~min}$, then resuspended in a staining solution, which contained $0.1 \%$ Triton X-100 and $500 \mathrm{mg} / \mathrm{ml}$ propidium iodide (PI). After incubation in the dark at room temperature for $30 \mathrm{~min}$, the cells were analysed by fluorescence-activated cell sorting (FACS) analysis.

Reverse transcription and polymerase chain reaction analysis $(R T-P C R)$. 143B cells were seeded in T25 flasks and treated with the indicated infection rates of AdBMP9 for $48 \mathrm{~h}$. Total RNA was extracted using the TRIzol reagent (Invitrogen, Carlsbad, CA, USA) and used to obtain cDNA templates by reverse transcription (RT). Then, the cDNAs were used as templates for determining the expression of target genes by semi-quantitative PCR (sqPCR), as described (36). The primers for each gene were as follows: GAPDH, F: 5'-CAAC GAATTTGGCTACAGCA-3', R: 5'-AGGGGAGATTCAGT GTGGTG-3'. BMP9, F: 5'-GCTCCGACTCTATGTCTCC TGT-3', R: 5'-CCAGCTTATTTTTGCTCTTGGT-3'.

Western blotting. The detailed method was previously described (36). In brief, subconfluent 143B cells were plated in 6-well plates and treated with the indicated concentrations of ATRA and/or AdBMP9 and/or SB203580. At the chosen timepoints, the cells were washed with ice-cold PBS and lysed with $300 \mu 1$ of lysis buffer. Then, the lysates were boiled for $10 \mathrm{~min}$. Total proteins were separated by SDS-PAGE and then transferred to polyvinylidene difluoride (PVDF) membranes. Membranes were blocked with bovine serum albumin (BSA) (5\%) at room temperature for $1 \mathrm{~h}$, and then blotted with primary antibodies. Finally, the bands corresponding to the targeted proteins were detected with the enhanced chemiluminescence method (ECL, substrate no. 34095; Thermo Fisher Scientific, USA).

Alkaline phosphatase (ALP) activity assay. ALP activity was assessed by a modified GreatEscape SEAPChemiluminescence assay (BD Clontech, Mountain View, CA, USA) as described previously (34). For the bioluminescence assays, each analysis was performed in triplicate, and the results were repeated in at least three independent experiments. ALP activity was normalized to total cellular protein concentration in each sample.

Statistical analysis. All the experiments were performed at least twice independently and the results were repeated in triplicate. Statistical analysis was performed using the GraphPad Prism 5 software (La Jolla, CA, USA). All data are represented as the mean \pm SD. Statistical significance between two groups 


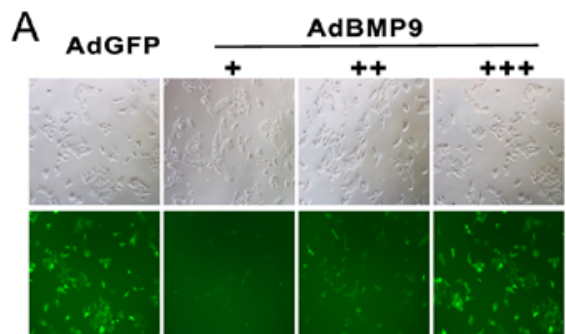

C

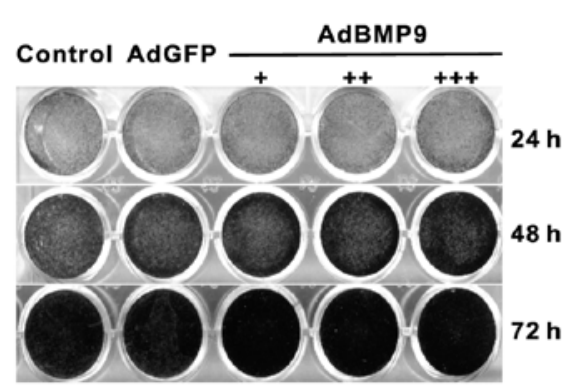

E

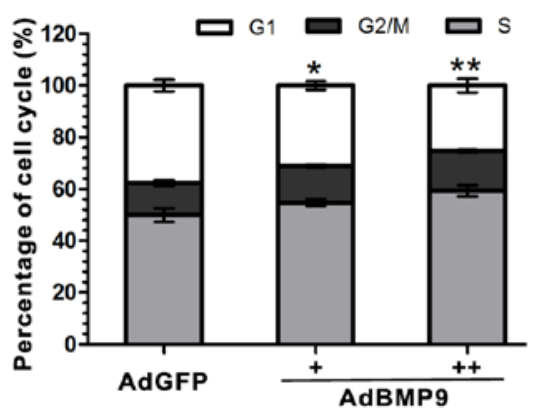

B
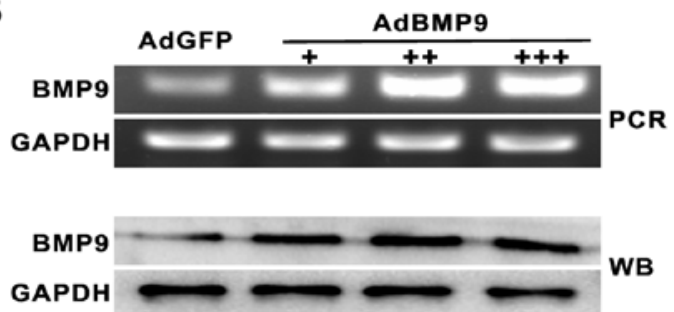

D

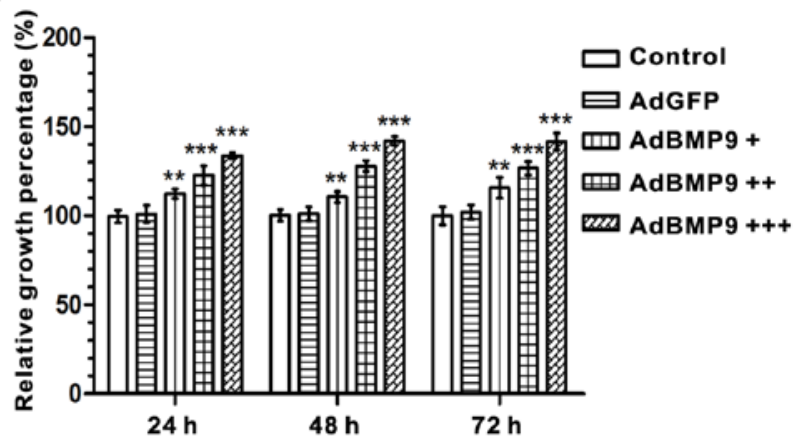

F

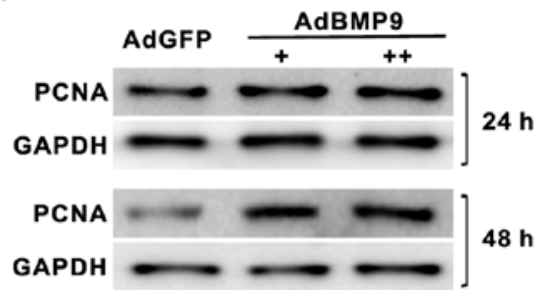

Figure 1. Effect of BMP9 on the proliferation of 143B cells. (A) AdBMP9 effectively transfected into 143B cells. 143B cells were infected with AdBMP9 and AdGFP. The GFP signal was detected under a fluorescence microscope (x100) $24 \mathrm{~h}$ after infection. (B) AdBMP9 effectively increased the expression of BMP9 $24 \mathrm{~h}$ after infection, as detected by semi-quantitative PCR (sqPCR) (top) and western blotting assay (bottom). (C) Crystal violet assays showed that BMP9 promoted proliferation in 143B cells. (D) Quantitative results of the crystal violet assay in $143 \mathrm{~B}$ cells $\left(^{* *} \mathrm{p}<0.01 \mathrm{vs}\right.$. the control group; ${ }^{* * * *} \mathrm{p}<0.001$ vs. the control group). (E) Quantitative results of cell cycle assay showed that BMP9 decreased the G1 phase arrest of $143 \mathrm{~B}$ cells $48 \mathrm{~h}$ after infection ( $\mathrm{*}<0.05$ vs. the AdGFP group; ${ }^{* *}$ p $<0.01$ vs. the AdGFP group). (F) Western blotting assays showed that the protein level of PCNA was affected by BMP9 in 143B cells. GAPDH was used as loading control. All assays were performed in triplicate. +, ++ and +++ refer to the infection rates of AdBMP9; PCNA, proliferating cell nuclear antigen.

was determined with Student's t-test. A value of $\mathrm{p}<0.05$ was considered to be statistically significant.

\section{Results}

BMP9 promotes the proliferation of OS cells. First, AdBMP9 infection was identified in human OS 143B cells (Fig. 1A and B). Then, to investigate the effect of BMP9 on 143B cell proliferation, cells were infected with AdBMP9 for 24, 48 or $72 \mathrm{~h}$ in 24 -well plates. The cell viability was assessed by crystal violet assay: the results showed that BMP9 promoted the proliferation of $143 \mathrm{~B}$ cells $(\mathrm{p}<0.01)$ (Fig. $1 \mathrm{C}$ and D). We next examined the cell cycle distribution of $143 \mathrm{~B}$ cells overexpressing BMP9 by flow cytometry; the results showed that BMP9 decreased the percentage of cells in G1 phase compared to the control group (Fig. 1E). The protein level of proliferating cell nuclear antigen (PCNA), which plays a key role in the cell cycle (37), was also significantly increased (Fig. 1F). These results suggested that BMP9 overexpression promoted the proliferation of 143B cells in vitro.
BMP9 failed to induce osteogenic differentiation of OS cells. To investigate the osteogenic differentiation activity of BMP9 in 143B cells, the expression level of osteogenesis-related markers was examined by western blotting and ALP activity assays. The data showed that the protein level of markers of both early osteogenic differentiation (such as the transcription factors Dlx-5, Runx-2) and late osteogenesis (such as OCN and $\mathrm{OPN}$ ) in 143B cells did not significantly change between the AdBMP9 and AdGFP groups (Fig. 2A and B). Similar results were obtained in the analysis of ALP activity, an early maker of osteogenesis (38) (Fig. 2C). These results demonstrated that BMP9 could not induce osteogenic differentiation of 143B cells in vitro.

$B M P 9$ inhibits the proliferation of $O S$ cells in the presence of ATRA. We next investigated the combined effect of BMP9 and ATRA on the proliferation of 143B cells. It has been reported that the proliferation of 143B cells is inhibited by ATRA in a concentration and time-dependent manner; the effective ATRA concentration may be $5,10,20,40$ or $80 \mu \mathrm{M}$ (29). At first, 20 and 


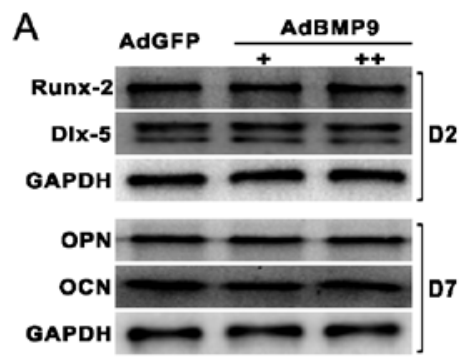

B

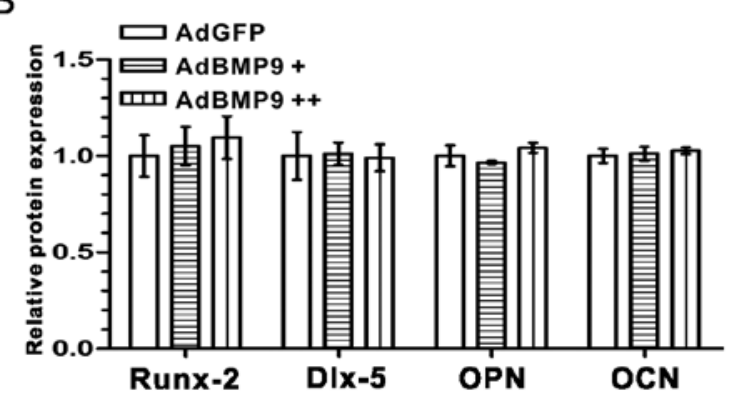

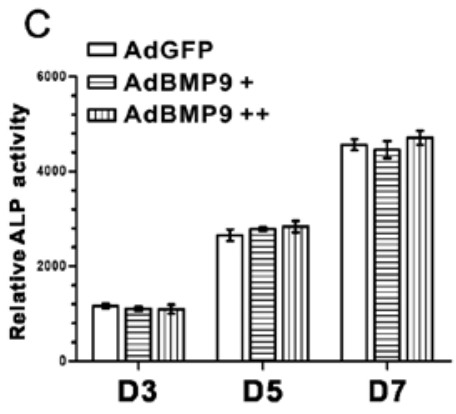

OCN

Figure 2. Effect of BMP9 on osteogenic differentiation in 143B cells. (A) Western blotting assays showed that the protein level of Dlx-5, Runx-2, OCN and OPN was not affected by BMP9. GAPDH was used as loading control. (B) Quantification of the western blotting results. (C) Quantitative ALP activity assay showed that the ALP activity was not affected by BMP9 in 143B cells. All assays were performed in triplicate. +,++ and +++ refer to the infection rates of AdBMP9.

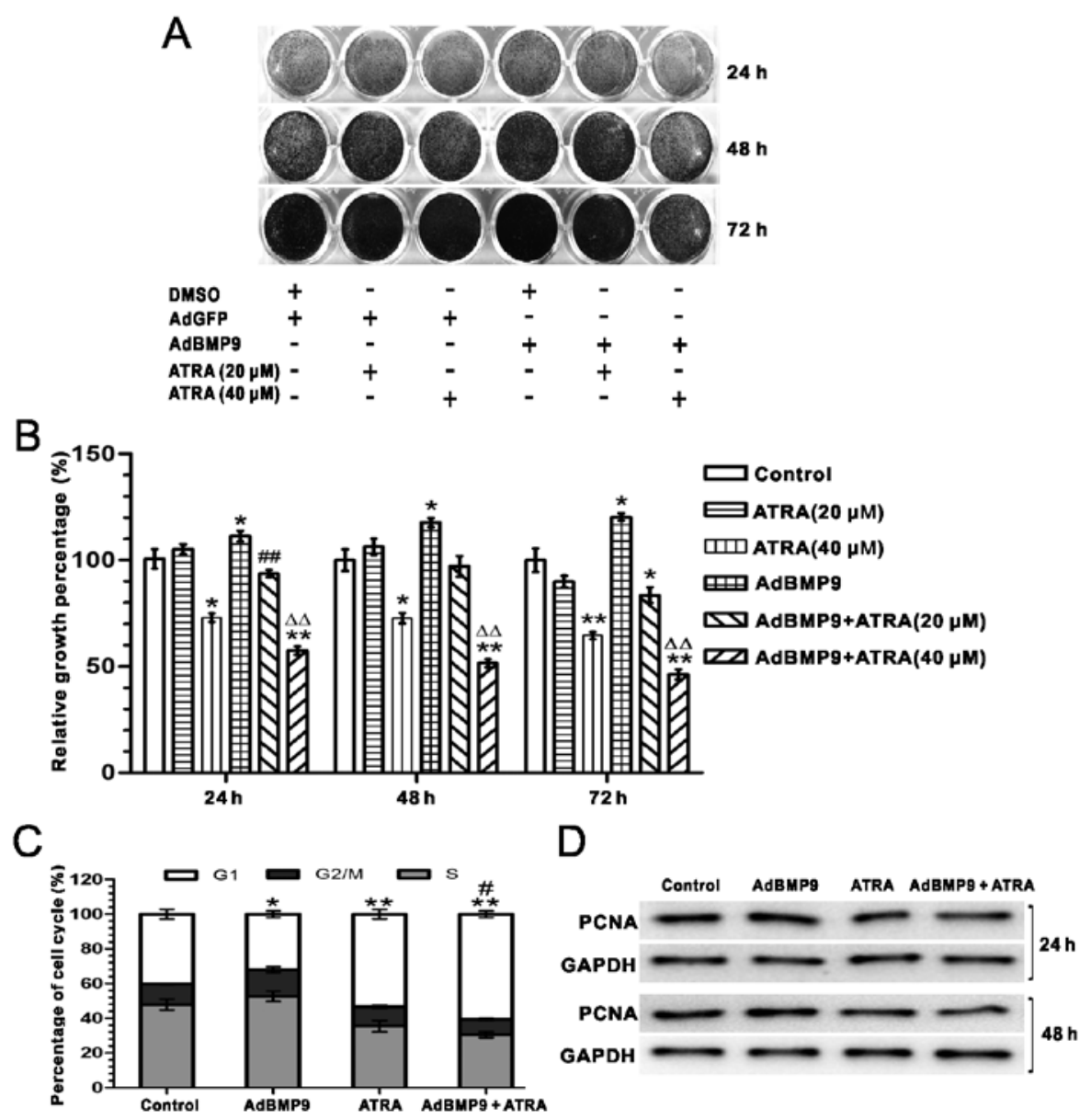

Figure 3. Effect of BMP9 on proliferation in the presence of ATRA in 143B cells. (A) Crystal violet assay showing the combined effects of BMP9 and ATRA on the proliferation of 143B cells. (B) Quantitative results of the crystal violet assay in $143 \mathrm{~B}$ cells. ( $\mathrm{p}<0.05$ vs. control group. ${ }^{* *} \mathrm{p}<0.01$ vs. control group; ${ }^{\# \#} \mathrm{p}<0.01$ vs. the ATRA $(20 \mu \mathrm{M})$ group; ${ }^{\Delta \Delta} \mathrm{p}<0.01$ vs. the ATRA $(40 \mu \mathrm{M})$ group). (C) Quantitative results of the cell cycle assay. The results showed that after $48 \mathrm{~h}, \mathrm{BMP} 9$ overexpression enhanced the effects of ATRA in 143B cells, increasing the fraction of cells arrested in the G1 phase of the cell cycle ("p $<0.05 \mathrm{vs}$. the control group; ${ }^{* *} \mathrm{p}<0.01$ vs. the control group; ${ }^{*} \mathrm{p}<0.05$ vs. the ATRA group). (D) Western blot assays showed that the protein level of PCNA was affected by concomitant treatment with BMP9 and ATRA in 143B cells. GAPDH was used as loading control. All assays were performed in triplicate. Cells treated with DMSO + AdGFP constituted the control group; PCNA, proliferating cell nuclear antigen. 


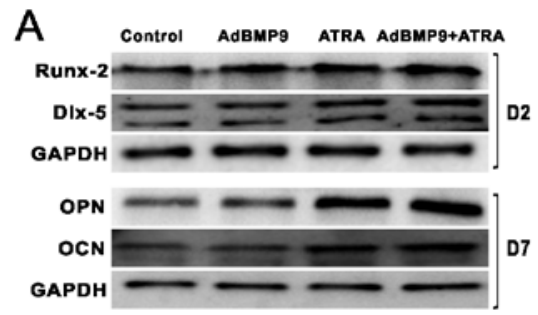

B

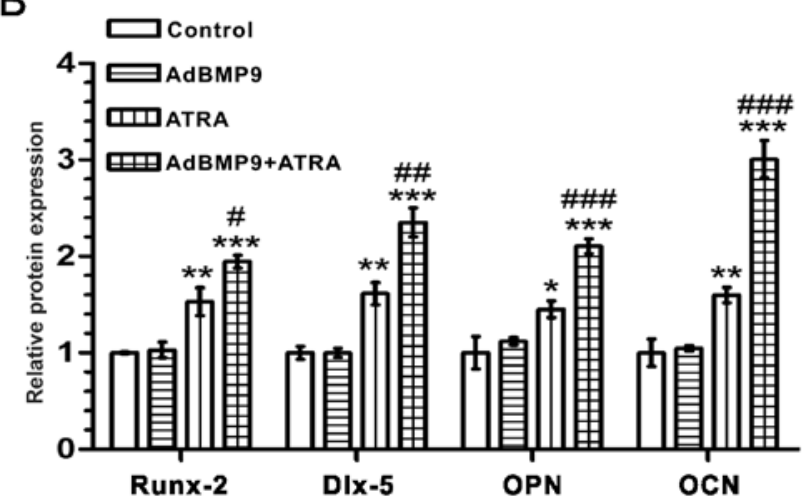

Figure 4. Effect of BMP9 on osteogenic differentiation in the presence of ATRA in 143B cells. (A) Western blot assays showed the protein level of Dlx-5, Runx-2, OCN and OPN were affected by concomitant treatment of 143B cells with BMP9 and ATRA. GAPDH was used as loading control. (B) Quantification of the western blotting results. (C) Quantitative ALP activity assay showed that the ALP activity was affected by the concomitant treatment of 143B cells with BMP9 and ATRA. All assays were performed in triplicate. Cells treated with DMSO + AdGFP constituted the control group. "p $<0.05$ vs. the control group; ${ }^{* *} \mathrm{p}<0.01$ vs. the control group; ${ }^{* * *} \mathrm{p}<0.001$ vs. the control group; ${ }^{\#} \mathrm{p}<0.05$ vs. the ATRA group; ${ }^{\# \#} \mathrm{p}<0.01$ vs. the ATRA group; ${ }^{\# \# \# ~} \mathrm{p}<0.001$ vs. the ATRA group.

$40 \mu \mathrm{M}$ were chosen as the candidate concentrations of ATRA: the crystal violet assay showed that there was no significant change in proliferation upon treatment with $20 \mu \mathrm{M}$ ATRA; however, the proliferation of 143B cells was significantly inhibited upon treatment with $40 \mu \mathrm{M}$ ATRA (p<0.05) (Fig. 3A and $\mathrm{B}$ ). This concentration was therefore used in the next steps of this study, together with the infection rate of ++ for AdBMP9.

A crystal violet assay suggested that BMP9 overexpression enhanced the anti-proliferative effect of ATRA $(p<0.01)$ (Fig. 3A and B). Likewise, cell cycle analysis showed that BMP9 overexpression enhanced the effects of ATRA-induced cell cycle arrest in G1 phase in 143B cells (Fig. 3C). A similar result was found by western blotting assays detecting the expression of PCNA (Fig. 3D). These results suggested that BMP9 could inhibit the proliferation of 143B cells in the presence of ATRA in vitro.

BMP9 induces the osteogenic differentiation of OS cells in the presence of ATRA. Then, we studied the osteogenic differentiation activity of BMP9 on OS cells in the presence of ATRA in vitro. The expression level of osteogenesis-related markers was analysed in 143B cells. Osteocalcin (OCN) expression was unchanged in 143B cells treated with BMP9, increased onehalf-fold in cells treated with ATRA ( $\mathrm{p}<0.01$, vs. the control group), and increased more than three-fold when the cells were treated with BMP9 and ATRA ( $<0.001$, vs. the control group) (Fig. 4A and B). Other osteogenesis-related markers (such as Dlx-5, Runx-2, OPN and ALP activity) showed similar changes (Fig. 4). These data indicated that BMP9 could induce the osteogenic differentiation of $143 \mathrm{~B}$ cells in the presence of ATRA in vitro.

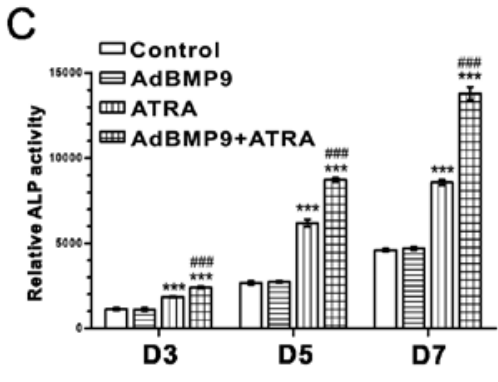


A

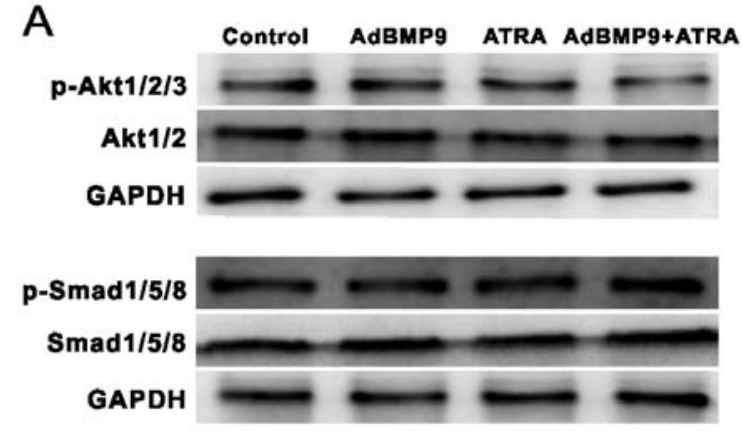

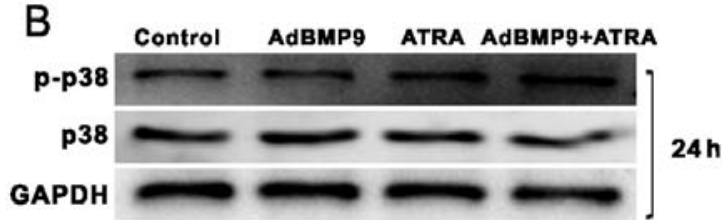

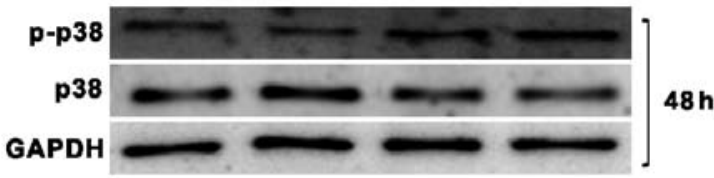

C

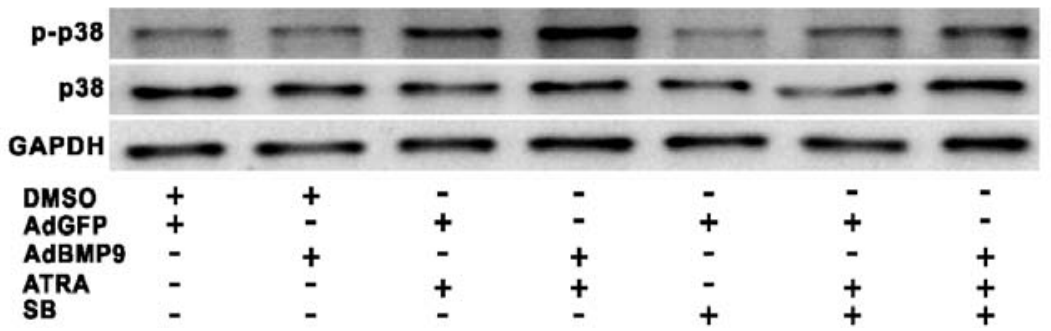

Figure 5. BMP9 activates the p38 MAPK pathway in the presence of ATRA, in 143B cells. (A) Western blot assays showing the protein levels of Smad1/5/8, phosphorylated Smad1/5/8 (p-Smad1/5/8), Akt1/2/3, phosphorylated Akt1/2/3 (p-Akt1/2/3) upon concomitant treatment with BMP9 and ATRA for 48 h in 143B cells. (B) Western blot assays showing the protein level of p38 and phosphorylated p38 (p-p38) upon concomitant treatment with BMP9 and ATRA in 143B cells. (C) Western blot assays showing the protein level of p38 and phosphorylated p38 (p-p38) upon concomitant treatment with BMP9 and ATRA and SB for $48 \mathrm{~h}$ in 143B cells. GAPDH was used as loading control. All assays were performed in triplicate. Cells treated with DMSO + AdGFP constituted the control group, SB, p38 inhibitor SB203580, $0.4 \mu \mathrm{M}$.

implied in tumourigenesis (7-13,16,19-22). The proliferative effect of BMP9 on tumours varies considerably. BMP9 promotes the proliferation of ovarian cancer cells (21) and hepatocellular carcinoma cells (22), but it inhibits the proliferation of colon cancer cells (19), breast cancer cells (20) and gastric cancer cells (16). However, the proliferative effect of BMP9 on OS is unclear. Li et al reported that BMP9 promoted human OS cell proliferation and tumour growth, possibly through the Notch signalling pathway (40). In contrast, Lv et al reported that BMP9 inhibited the growth of OS cells through the Wnt/ $\beta$-catenin pathway (41). Our study suggested that BMP9 promoted proliferation (Fig. 1) and failed to induce osteogenic differentiation in human OS 143B cells (Fig. 2). It has been suggested that the tight link between cell proliferation and differentiation is often compromised in cancer cells, and the inhibition of proliferation can result from the induction of differentiation (42-47). The modulation of the activity of transcription factors can promote differentiation and inhibit proliferation, and it has been used in the treatment of leukaemia (46). Similarly, the overexpression of CDK inhibitors prevents proliferation and simultaneously induces differentiation in a variety of tumour cells (42-44). Therefore, the restoration of the osteogenic ability of BMP9 in OS cells may contribute to the treatment of OS.

ATRA promotes terminal differentiation of immature cells, including various types of cancer cells (25-30), and can promote osteogenic differentiation (29-33,48). Yang et al reported that ATRA could inhibit proliferation, induce apotosis and promote osteogenic differentiation in 143B cells, but the effect of apotosis induced by ATRA is not obvious. Therefore, they concluded that ATRA inhibits the proliferation of OS by inducing osteogenesis (29). In this study, it is comfirmed that BMP9 could not induce osteogenic differentiation alone in
143B cells (Fig. 2) (8,9,11-13). However, BMP9 induced osteogenic differentiation in 143B cells in the presence of ATRA $(p<0.001)$ (Fig. 4). The effect of the combination of BMP9 and ATRA on the inhibition of proliferation of 143B cells was more significant than that of ATRA alone $(\mathrm{p}<0.01)$ (Fig. 3). This indicates that ATRA can restore the osteogenic ability of BMP9, thus inhibiting the proliferation of 143B cells. BMP9 signals through the canonical BMP/Smad pathway. BMP9 binds to type II or type I BMP receptors (BMPRII or BMPRI), phosphorylates Smad1/5/8 and forms a complex with Smad4, followed by translocation to the nucleus and regulation of downstream targets $(18,38)$. However, this study suggested that ATRA alone or ATRA combined with BMP9 groups failed to activate the Smad1/5/8 signalling (Fig. 5A), indicating that the osteogenesis induced by BMP9 in the presence of ATRA may not be mediated through the canonical BMP/Smad pathway in 143B cells. The combination of ATRA and BMP9 also failed to activate the Akt1/2/3 signalling (Fig. 5A). BMP9 can exert its function through the non-canonical BMP/Smad pathway, involving p38 MAPK and PI3K/Akt $(38,49)$. Thus, we surmised that p38 MAPK might play an important role in this phenomenon.

It has been reported that p38 MAPK is involved in cell differentiation, proliferation, apoptosis, metastasis and autophagy (19,50-53). Activation of p38 MAPK is essential for BMP9-induced osteogenesis in mesenchymal progenitor cells $(49,54,55)$. However, BMP9 failed to activate p38 MAPK in 143B cells (Fig. 5B) indicating that the inactivation of p38 MAPK might be the cause for the ineffectiveness of BMP9 to activate osteogenesis in OS cells. This study further suggests that ATRA activated p38 MAPK, and the concomitant treatment with ATRA and BMP9 significantly enhanced this effect $(p<0.01)$ (Fig. 5B). The osteogenic differentiation 


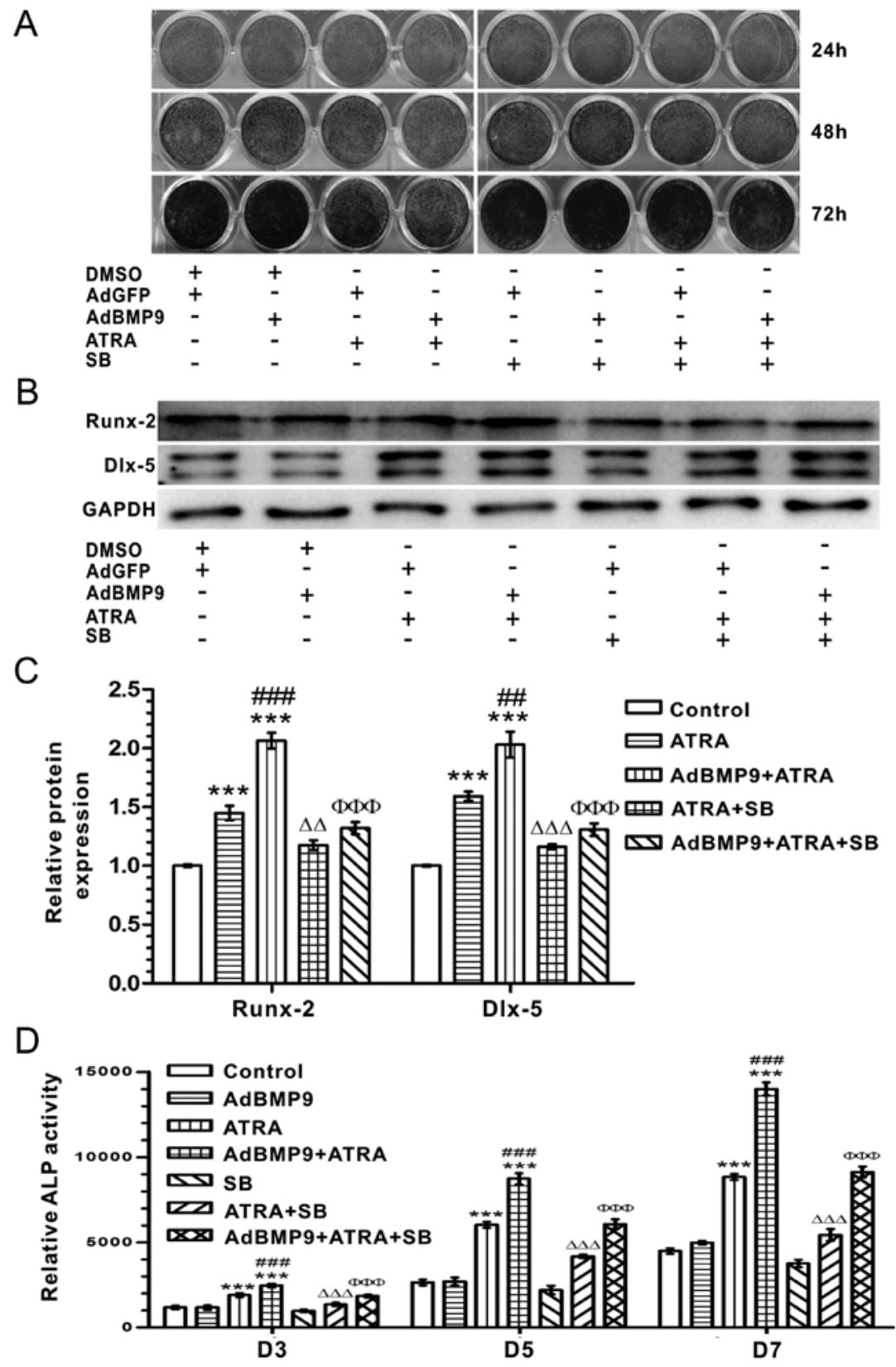

Figure 6. BMP9 affects 143B cells by activating the p38 MAPK pathway in the presence of ATRA. (A) Crystal violet assay showing the effect of the concomitant treatment with BMP9, ATRA and SB on the proliferation of 143B cells. (B) Western blot assays showed that the protein levels of Dlx-5 and Runx-2 were affected by concomitant treatment with BMP9, ATRA and SB for 2 days in 143B cells. GAPDH was used as loading control. (C) Quantification of the western blotting results. (D) Quantitative ALP activity assays showed that the ALP activity was affected by concomitant treatment with BMP9, ATRA and SB in 143B cells. All assays were performed in triplicate. Cells treated with DMSO + AdGFP constituted the control group. ${ }^{* * *}$ p $<0.001$ vs. the control group; ${ }^{\# \#} \mathrm{p}<0.01$ vs. the ATRA group; ${ }^{\# \# \#} \mathrm{p}<0.001$ vs. the ATRA group; ${ }^{\Delta \Delta} \mathrm{p}<0.01$ vs. the ATRA group; ${ }^{\Delta \Delta \Delta} \mathrm{p}<0.001$ vs. the ATRA group; ${ }^{\phi \phi \phi} \mathrm{p}<0.001$ vs. the AdBMP9 + ATRA group. SB, p38 inhibitor SB203580, $0.4 \mu \mathrm{M}$.

and proliferation inhibition effects of ATRA alone or ATRA combined with BMP9 groups were inhibited by a p38 MAPK inhibitor (SB203580) (Fig. 6). These results indicate that ATRA restored osteogenic ability of BMP9 in 143B cells, probably through the reactivation of p38 MAPK.

In conclusion, this study suggests that the proliferative effect of BMP9 on human OS 143B cells is related to a failure in osteogenic differentiation. ATRA could restore the osteogenic ability of BMP9 in 143B cells, and the combination of ATRA and BMP9 generated a more significant anti-proliferative effect than ATRA alone. This result may be due to the reactivation of the p38 MAPK pathway.

\section{Acknowledgements}

We would like to thank Dr T.C. He (University of Chicago Medical Center, USA) for generously providing all recombinant adenoviruses. This study was supported by research grants from the Natural Science Foundation of China (NSFC 81572226 to B.C.H. and 81601895 to R.L.).

\section{References}

1. Helman LJ and Meltzer P: Mechanisms of sarcoma development. Nat Rev Cancer 3: 685-694, 2003. 
2. Mirabello L, Troisi RJ and Savage SA: Osteosarcoma incidence and survival rates from 1973 to 2004: Data from the Surveillance, Epidemiology, and End Results Program. Cancer 115: 1531-1543, 2009.

3. Whelan JS, Bielack SS, Marina N, Smeland S, Jovic G, Hook JM, Krailo M, Anninga J, Butterfass-Bahloul T, Böhling T, et al EURAMOS collaborators: EURAMOS-1, an international randomised study for osteosarcoma: Results from pre-randomisation treatment. Ann Oncol 26: 407-414, 2015.

4. Wang LL: Biology of osteogenic sarcoma. Cancer J 11: 294-305, 2005.

5. Isakoff MS, Bielack SS, Meltzer P and Gorlick R: Osteosarcoma: Current treatment and a collaborative pathway to success. J Clin Oncol 33: 3029-3035, 2015.

6. Dai X, Ma W, He X and Jha RK: Review of therapeutic strategies for osteosarcoma, chondrosarcoma, and Ewing's sarcoma. Med Sci Monit 17: RA177-RA190, 2011.

7. Thomas D and Kansara M: Epigenetic modifications in osteogenic differentiation and transformation. J Cell Biochem 98: 757-769, 2006.

8. Haydon RC, Luu HH and He TC: Osteosarcoma and osteoblastic differentiation: A new perspective on oncogenesis. Clin Orthop Relat Res 454: 237-246, 2007.

9. Tang N, Song WX, Luo J, Haydon RC and He TC: Osteosarcoma development and stem cell differentiation. Clin Orthop Relat Res 466: 2114-2130, 2008

10. Luo X, Chen J, Song WX, Tang N, Luo J, Deng ZL, Sharff KA, $\mathrm{He} \mathrm{G}, \mathrm{Bi}$ Y, He BC, et al: Osteogenic BMPs promote tumor growth of human osteosarcomas that harbor differentiation defects. Lab Invest 88: 1264-1277, 2008.

11. Wang J, Zhang $\mathrm{H}$, Zhang W, Huang E, Wang N, Wu N, Wen S, Chen X, Liao Z, Deng F, et al: Bone morphogenetic protein- 9 effectively induces osteo/odontoblastic differentiation of the reversibly immortalized stem cells of dental apical papilla. Stem Cells Dev 23: 1405-1416, 2014

12. Li Y, Wagner ER, Yan Z, Wang Z, Luther G, Jiang W, Ye J, Wei Q, Wang J, Zhao L, et al: The calcium-binding protein S100A6 accelerates human osteosarcoma growth by promoting cell proliferation and inhibiting osteogenic differentiation. Cell Physiol Biochem 37: 2375-2392, 2015.

13. Yang R, Piperdi S, Zhang Y, Zhu Z, Neophytou N, Hoang BH Mason G, Geller D, Dorfman H, Meyers PA, et al: Transcriptional profiling identifies the signaling axes of IGF and transforming growth factor- $\beta$ as involved in the pathogenesis of osteosarcoma. Clin Orthop Relat Res 474: 178-189, 2016

14. Sánchez-Duffhues G, Hiepen C, Knaus P and Ten Dijke P: Bone morphogenetic protein signaling in bone homeostasis. Bone 80 : 43-59, 2015.

15. Chen D, Zhao M, Harris SE and Mi Z: Signal transduction and biological functions of bone morphogenetic proteins. Front Biosci 9: 349-358, 2004.

16. Duan L, Ye L, Wu R, Wang H, Li X, Li H, Yuan S, Zha H, Sun H Zhang Y, et al: Inactivation of the phosphatidylinositol 3-kinase/ Akt pathway is involved in BMP9-mediated tumor-suppressive effects in gastric cancer cells. J Cell Biochem 116: 1080-1089, 2015.

17. Xiang L, Liang C, Zhen-Yong K, Liang-Jun Y and ZhongLiang D: BMP9-induced osteogenetic differentiation and bone formation of muscle-derived stem cells. J Biomed Biotechnol 2012: 610952, 2012

18. Wang JH, Liu YZ, Yin LJ, Chen L, Huang J, Liu Y, Zhang RX, Zhou LY, Yang QJ, Luo JY, et al: BMP9 and COX-2 form an important regulatory loop in BMP9-induced osteogenic differentiation of mesenchymal stem cells. Bone 57: 311-321, 2013.

19. Yuan SX, Wang DX, Wu QX, Ren CM, Li Y, Chen QZ, Zeng YH, Shao Y, Yang JQ, Bai Y, et al: BMP9/p38 MAPK is essential for the antiproliferative effect of resveratrol on human colon cancer. Oncol Rep 35: 939-947, 2016.

20. Holtzhausen A, Golzio C, How T, Lee YH, Schiemann WP, Katsanis N and Blobe GC: Novel bone morphogenetic protein signaling through Smad 2 and Smad3 to regulate cancer progression and development. FASEB J 28: 1248-1267, 2014.

21. Herrera B, van Dinther M, Ten Dijke P and Inman GJ: Autocrine bone morphogenetic protein-9 signals through activin receptorlike kinase-2/Smad1/Smad4 to promote ovarian cancer cell proliferation. Cancer Res 69: 9254-9262, 2009.

22. Herrera B, García-Álvaro M, Cruz S, Walsh P, Fernández M, Roncero C, Fabregat I, Sánchez A and Inman GJ: BMP9 isa proliferative and survival factor for human hepatocellular carcinoma cells. PloS One 8: e69535, 2013.
23. Wu LZ, Chaudhary SC, Atigadda VR, Belyaeva OV, Harville SR, Elmets CA, Muccio DD, Athar M, Kedishvili NY and Retinoid X: Receptor agonists upregulate genes Responsible for the biosynthesis of all-trans-retinoic acid in human epidermis. PLoS One 11: e01535562016, 2016.

24. Dilworth FJ and Chambon P: Nuclear receptors coordinate the activities of chromatin remodeling complexes and coactivators to facilitate initiation of transcription. Oncogene 20: 3047-3054, 2001.

25. Atashrazm F, Lowenthal RM, Dickinson JL, Holloway AF and Woods GM: Fucoidan enhances the therapeutic potential of arsenic trioxide and all-trans retinoic acid in acute promyelocytic leukemia, in vitro and in vivo. Oncotarget 7: 46028-46041, 2016.

26. Silvis AM, McCormick ML, Spitz DR and Kiningham KK: Redox balance influences differentiation status of neuroblastoma in the presence of all-trans retinoic acid. Redox Biol 7: 88-96, 2016.

27. Yan Y, Li Z, Xu X, Chen C, Wei W, Fan M, Chen X, Li JJ, Wang Y and Huang J: All-trans retinoic acids induce differentiation and sensitize a radioresistant breast cancer cells to chemotherapy. BMC Complement Altern Med 16: 113-123, 2016.

28. Amann PM, Czaja K, Bazhin AV, Rühl R, Skazik C, Heise R, Marquardt Y, Eichmüller SB, Merk HF and Baron JM: Knockdown of lecithin retinol acyltransferase increases all-trans retinoic acid levels and restores retinoid sensitivity in malignant melanoma cells. Exp Dermatol 23: 832-837, 2014.

29. Yang QJ, Zhou LY, Mu YQ, Zhou QX, Luo JY, Cheng L, Deng ZL, He TC, Haydon RC and He BC: All-trans retinoic acid inhibits tumor growth of human osteosarcoma by activating Smad signaling-induced osteogenic differentiation. Int J Oncol 41: $153-160,2012$.

30. Zhang L, Zhou Q, Zhang N, Li W, Ying M, Ding W, Yang B and He Q: E2F1 impairs all-trans retinoic acid-induced osteogenic differentiation of osteosarcoma via promoting ubiquitinationmediated degradation of RAR $\alpha$. Cell Cycle 13: 1277-1287, 2014.

31. Luo P, Yang X, Ying M, Chaudhry P, Wang A, Shimada H, May WA, Adams GB, Mock D, Triche TJ, et al: Retinoidsuppressed phosphorylation of RARalpha mediates the differentiation pathway of osteosarcoma cells. Oncogene 29: 2772-2783, 2010

32. Ying M, Zhang L, Zhou Q, Shao X, Cao J, Zhang N, Li W, Zhu H, Yang $\mathrm{B}$ and $\mathrm{He} \mathrm{Q}$ : The E3 ubiquitin protein ligase MDM2 dictates all-trans retinoic acid-induced osteoblastic differentiation of osteosarcoma cells by modulating the degradation of RAR $\alpha$. Oncogene 35: 4358-4367, 2016.

33. Liu Y, Liu Y, Zhang R, Wang X, Huang F, Yan Z, Nie M, Huang J, Wang Y, Wang Y, et al: All-trans retinoic acid modulates bone morphogenic protein 9-induced osteogenesis and adipogenesis of preadipocytes through BMP/Smad and $\mathrm{Wnt} / \beta$-catenin signaling pathways. Int J Biochem Cell Biol 47: 47-56, 2014.

34. Tang N, Song WX, Luo J, Luo X, Chen J, Sharff KA, Bi Y, He BC, Huang JY, Zhu GH, et al: BMP-9-induced osteogenic differentiation of mesenchymal progenitors requires functional canonical Wnt/beta-catenin signalling. J Cell Mol Med 13B: 2448-2464, 2009.

35. He BC, Chen L, Zuo GW, Zhang W, Bi Y, Huang J, Wang Y, Jiang W, Luo Q, Shi Q, et al: Synergistic antitumor effect of the activated PPARgamma and retinoid receptors on human osteosarcoma. Clin Cancer Res 16: 2235-2245, 2010.

36. Meng ZJ, Wu N, Liu Y, Shu KJ, Zou X, Zhang RX, Pi CJ, He BC, Ke ZY, Chen L, et al: Evodiamine inhibits the proliferation of human osteosarcoma cells by blocking PI3K/Akt signaling. Oncol Rep 34: 1388-1396, 2015.

37. Strzalka W and Ziemienowicz A: Proliferating cell nuclear antigen (PCNA): A key factor in DNA replication and cell cycle regulation. Ann Bot 107: 1127-1140, 2011.

38. Huang J, Yuan SX, Wang DX, Wu QX, Wang X, Pi CJ, Zou X, Chen L, Ying LJ, Wu K, et al: The role of COX-2 in mediating the effect of PTEN on BMP9 induced osteogenic differentiation in mouse embryonic fibroblasts. Biomaterials 35: 9649-9659, 2014.

39. Herrera B, Dooley S and Breitkopf-Heinlein K: Potential roles of bone morphogenetic protein (BMP)-9 in human liver diseases. Int J Mol Sci 15: 5199-5220, 2014.

40. Li R, Zhang W, Cui J, Shui W, Yin L, Wang Y, Zhang H, Wang N, Wu N, Nan G, et al: Targeting BMP9-promoted human osteosarcoma growth by inactivation of notch signaling. Curr Cancer Drug Targets 14: 274-285, 2014. 
41. Lv Z, Wang C, Yuan T, Liu Y, Song T, Liu Y, Chen C, Yang M, Tang Z, Shi Q, et al: Bone morphogenetic protein 9 regulates tumor growth of osteosarcoma cells through the Wnt/ $\beta$-catenin pathway. Oncol Rep 31: 989-994, 2014.

42. Kranenburg O, Scharnhorst V, Van der Eb AJ and Zantema A: Inhibition of cyclin-dependent kinase activity triggers neuronal differentiation of mouse neuroblastoma cells. J Cell Biol 131: 227-234, 1995

43. Adachi M, Roussel MF, Havenith K and Sherr CJ: Features of macrophage differentiation induced by p19INK4d, a specific inhibitor of cyclin D-dependent kinases. Blood 90: 126-137, 1997.

44. Matushansky I, Radparvar F and Skoultchi AI: Reprogramming leukemic cells to terminal differentiation by inhibiting specific cyclin-dependent kinases in G1. Proc Natl Acad Sci USA 97: 14317-14322, 2000.

45. Rosenbauer $\mathrm{F}$ and Tenen DG: Transcription factors in myeloid development: Balancing differentiation with transformation. Nat Rev Immunol 7: 105-117, 2007.

46. Hanahan D and Weinberg RA: Hallmarks of cancer: The next generation. Cell 144: 646-674, 2011

47. Ruijtenberg S and van den Heuvel S: Coordinating cell proliferation and differentiation: Antagonism between cell cycle regulators and cell type-specific gene expression. Cell Cycle 15 196-212, 2016

48. Zhang W, Deng ZL, Chen L, Zuo GW, Luo Q, Shi Q, Zhang BQ Wagner ER, Rastegar F, Kim SH, et al: Retinoic acids potentiate BMP9-induced osteogenic differentiation of mesenchymal progenitor cells. PLoS One 5: e11917, 2010.
49. Zhao Y, Song T, Wang W, Wang J, He J, Wu N, Tang M, He B and Luo J: P38 and ERK1/2 MAPKs act in opposition to regulate BMP9-induced osteogenic differentiation of mesenchymal progenitor cells. PLoS One 7: e43383, 2012.

50. Cuadrado A and Nebreda AR: Mechanisms and functions of $\mathrm{p} 38$ MAPK signalling. Biochem J 429: 403-417, 2010.

51. Cheng HL, Lin CW, Yang JS, Hsieh MJ, Yang SF and Lu KH: Zoledronate blocks geranylgeranylation not farnesylation to suppress human osteosarcoma U2OS cells metastasis by EMT via Rho A activation and FAK-inhibited JNK and p38 pathways. Oncotarget 7: 9742-9758, 2016.

52. Lv T, Wu Y, Mu C, Liu G, Yan M, Xu X, Wu H, Du J, Yu J and $\mathrm{Mu} \mathrm{J}$ : Insulin-like growth factor 1 promotes the proliferation and committed differentiation of human dental pulp stem cells through MAPK pathways. Arch Oral Biol 72: 116-123, 2016.

53. Liu J, Wang B, Huang P, Wang H, Xu K, Wang X, Xu L and Guo Z: Microcystin-LR promotes cell proliferation in the mice liver by activating Akt and p38/ERK/JNK cascades. Chemosphere 163: $14-21,2016$

54. Xu DJ, Zhao YZ, Wang J, He JW, Weng YG and Luo JY: Smads, p38 and ERK1/2 are involved in BMP9-induced osteogenic differentiation of $\mathrm{C} 3 \mathrm{H} 10 \mathrm{~T} 1 / 2$ mesenchymal stem cells. BMB Rep 45: 247-252, 2012.

55. Ye G, Li C, Xiang X, Chen C, Zhang R, Yang X, Yu X, Wang J, Wang L, Shi Q, et al: Bone morphogenetic protein-9 induces PDLSCs osteogenic differentiation through the ERK and p38 signal pathways. Int J Med Sci 11: 1065-1072, 2014. 